\title{
Structural control and physical properties of dianionic naphthalenediimide derivative
}

$\underline{\text { Ayumi Kawasaki }}^{1}$, Takashi Takeda ${ }^{2}$, Norihisa Hoshino ${ }^{2}$, Tomoyuki Akutagawa ${ }^{2}$

${ }^{1}$ Graduate school of engineering, Tohoku University, Sendai, Japan, ${ }^{2}$ Institute of Multidisciplinary Research for Advanced Materials, Tohoku University, Sendai, Japan

E-mail: a.kawa@mail.tagen.tohoku.ac.jp

Excellent $n$-type semiconducting property has been reported in naphthalenediimide (NDI) pi-framework. Since the performance of organic semiconductors is mainly dominated by the packing structure of each pi-molecule, precise control in the molecular assembly structures should be one of the important points of view to increase the device performance. Various types of molecular assembly structures can be designed by introducing ion pairing units into NDI pi-framework. Herein, we prepared phenylsulfonate substituted NDI derivatives of salts $\mathbf{1}, \mathbf{2}, \mathbf{3}$, and $\mathbf{4}$ by combining with two molar of alkylammonium cations and evaluated its physical properties.

Figure 1 showed the cyclic voltammetry (CV) curves of $2: 1$ salts $\mathbf{1}$ and $\mathbf{2}$ together with parent $\mathrm{Na}^{+}$salt. Since all salts exhibited the two-step reversible reduction waves around -0.43 and $-0.90 \mathrm{~V}$ in $\mathrm{DMF}$ vs $\mathrm{Ag} / \mathrm{AgCl}$, the counter cations did not affect the electron-accepting ability of phenylsulfonate substituted NDI pi-framework. The molecular assembly structures of salts $\mathbf{1}$ and $\mathbf{2}$ were evaluated by the powder X-ray diffraction (PXRD) technique (Figure 2). The PXRD pattern of salt $\mathbf{1}$ showed the sharp diffraction peaks due to high crystallinity. The crystal structure of salt $\mathbf{3}$ was obtained by the single crystal $X$-ray structural analysis. The cation-anion layer structure was elongated along the $c$ axis, whereas the one-dimensional pistacking structure of NDI core was observed along the a axis. Although the similar layered cation-anion packing structure and one-dimensional pi-stack were assumed in salt $\mathbf{1}$, the interlayer spacing $d_{001}$ of salt $\mathbf{1}$ was $22 \AA$ longer than that of salt $3\left(d_{001}=15 \AA\right)$. On the contrary, although the alternate cation-anion layer was kept in salt $\mathbf{2}$, in-plane periodicity was decreased by introducing two long $-\mathrm{C}_{12} \mathrm{H}_{25}$ chains due to broad PXRD pattern at high angle diffraction angle. In addition, single crystal $\mathrm{X}$-ray structure analysis of salt $\mathbf{4}$ revealed the formation of the two-dimensional herring-boron packing structure of NDI pi-frameworks. In summary, the molecular structure of counter cations affects the molecular assembly structures of NDI pi-framework, which can be easily modified by changing in the cation exchange processes.

a)

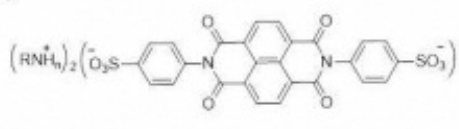

b)

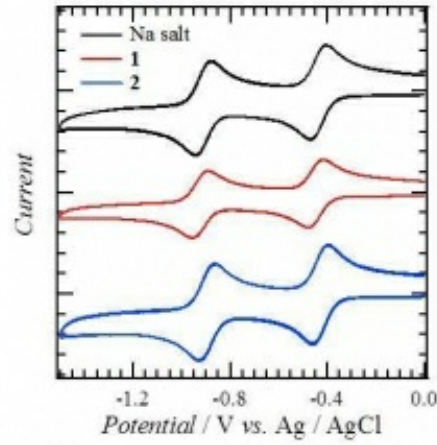

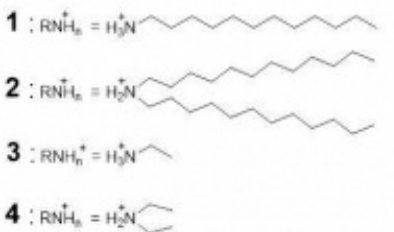

c)

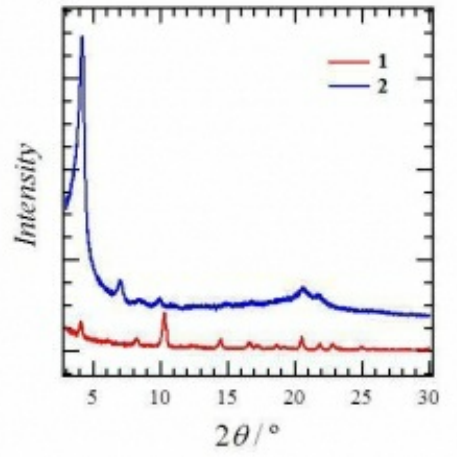

Figure a ) Salt 1-4, b) CV curves of each salts (in DMF / $\mathrm{TBABF}_{4}, \mathrm{WE}: \mathrm{GC}, \mathrm{RE}: \mathrm{Ag} / \mathrm{AgCl}$, $\mathrm{CE}: \mathrm{Pt}$, c ) PXRD pattern of salt 1 and $\mathbf{2}$ at $30^{\circ} \mathrm{C}$

Keywords: Naphthalenediimide, Organic Semiconductor, Molecular Assembly 